\title{
Soil treatment in flood areas: case of the road RP4209, in the province of Kenitra (Morocco)
}

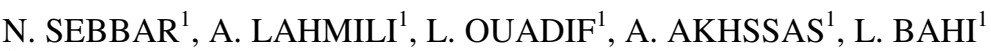 \\ ${ }^{1}$ Mohammed V University, Mohammadia School of Engineering, L3GIE, Rabat, MOROCCO.
}

\begin{abstract}
The Moroccan road network has a linear of more than $57,000 \mathrm{~km}$ divided into national, regional, provincial and unclassified roads. Population growth, the transport of goods and the mobility of people require the construction of new links between regions and the maintenance of existing ones. Indeed, several factors, such as rainfall and floods, can cause enormous damage to existing infrastructure. Among the most at-risk areas is the GHARB region, especially the province of Kénitra, represented in this study by Provincial Road RP4209, which includes flood-prone areas with abundant watercourses, associated with poor type of soil. To mitigate these phenomena and to try to reduce their impacts, in particular on the components of a road, one of the appropriate solutions remains the treatment of soils. This process is increasingly practiced in the construction industry, improves the geotechnical and mechanical characteristics of soils without having to go through the contribution of materials from other regions, all for the purpose of a valuation of materials in place by treatment with lime, different hydraulic binders, or industrial products, which will provide an economic and environmental gain
\end{abstract}

Résumé. Le réseau routier marocain compte un linéaire de plus de 57.000 kms réparties en routes nationales, régionales, provinciales et routes non classées. La croissance démographique, le transport de marchandises ainsi que la mobilité des personnes imposent la construction de nouvelles liaisons entre régions et l'entretien de celles déjà existantes. En effet, plusieurs facteurs, tels que les précipitations et les inondations, peuvent engendrer d'énormes dégâts sur l'infrastructure existante. Parmi les régions les plus à risques, on trouve la région du GHARB en particulier la province de Kénitra, représentée dans cette étude par la route provinciale RP4209, qui regroupe des zones inondables avec l'abondance des cours d'eau, associée à la mauvaise nature du sol. Pour pallier à ces phénomènes et tenter de diminuer leurs impacts, en particulier sur les composantes d'une route, l'une des solutions appropriées demeure le traitement des sols. Ce procédé qu'est de plus en plus pratiqué dans le milieu du BTP, améliore les caractéristiques géotechniques et mécaniques des sols sans avoir à passer par l'apport des matériaux d'autres régions, tout cela dans le but d'une valorisation des matériaux en place par un traitement à la chaux, aux différents liants hydrauliques, ou aux produits industriels, ce qui procurera un gain économique ainsi qu'environnemental [1].

\section{Introduction}

De nos jours, le transport est devenu une activité primordiale pour le développement de tout pays. Il va de soi, que ce nombre en augmentation quotidienne a, en plus de ses avantages incontestables sur le train de vie actuel des individus, aussi des impacts néfastes que ce soit sur notre environnement ou sur nos infrastructures. La qualité des chaussées dépend de plusieurs facteurs, entre autre la portance des sols supports. Une faible portance de la plateforme provoquera des déformations qui vont remonter jusqu'à la chaussée et causeront des orniérages et fissurations, parmi d'autres. Cette mauvaise portance est due essentiellement aux caractéristiques de la nature du sol.

L'approche habituelle dans ces cas, c'est d'enlever le mauvais sol (argileux, dans notre cas) et de le substituer par un sol plus résistant et moins fins. Toutefois, cette solution demeure plus onéreuse.

Pour ces raisons économiques et aussi environnementales, le choix de la solution du traitement et surtout avec de la chaux vive, demeure la plus adéquate pour ce type de sols.

La chaux vive permet de réaliser des modifications sur l'état hydrique du sol par la diminution de la teneur en eau du mélange sol-liant, provoquée par une 
hydratation de la chaux vive et par vaporisation d'une quantité d'eau causée par cette hydratation. Ceci permettra la réduction de la sensibilité du sol à l'eau et aussi du cycle gonflement-retrait de ce sol [2].

Dans cet article, on se penchera en premier lieu, sur une caractérisation de la zone à étudier, par l'identification du sol via une étude géotechnique. Dans cette étude on insistera sur les essais caractérisant l'argilosité du sol (limite d'Atterberg, valeur au bleu de méthylène), tout en passant par une analyse granulométrique dans le but de déterminer la répartition dimensionnelle des grains dans notre sol. Ensuite on se focalisera sur les études de formulation du couple solliant. Cette étape consistera en l'obtention d'une meilleure portance.

\section{Cadre géographique et géologique}

Une caractérisation de la zone à étudier est importante dans le cadre de notre projet, pour nous permettre de situer les zones d'existence des matériaux à traiter, ce qui mènera à une classification géologique et géotechnique des différents matériaux de la zone d'étude, facilitant ainsi la détermination des différents sols favorables pour les divers traitements .

\subsection{Cadre géographique}

La plaine du Gharb (Figure 1) se situe dans la partie Nord-Ouest du royaume. Elle est limitée à l'Ouest par l'océan Atlantique, au nord par la région de TangerTétouan, à l'Est par les deux régions de Taza-Al Hoceima- Taounate et de Fès-Boulemane et au Sud par les régions de Meknès-Tafilalet et de Rabat-SaléZemmour-Zaër.

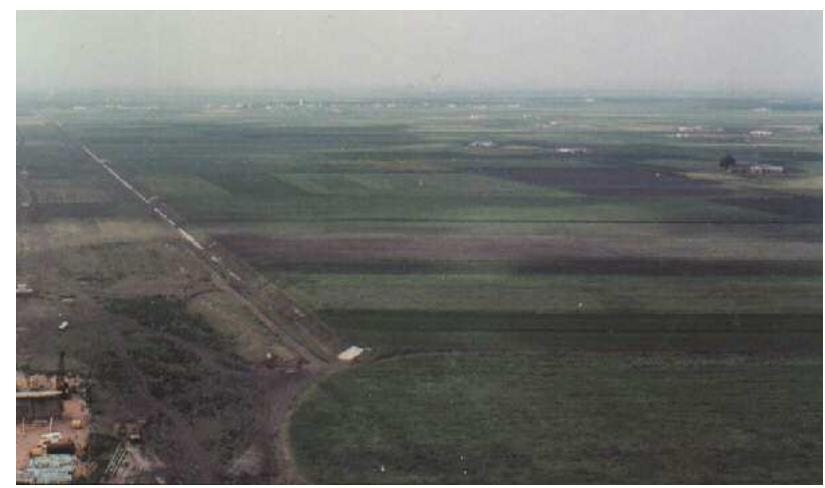

Fig. 1 : Vue générale de la plaine du Gharb (Ormvag) [3]

La région du Gharb bénéficie d'un climat méditerranéen, caractérisée par des hivers doux et humides et des étés chauds et secs où la moyenne des températures allant de $23^{\circ} \mathrm{C}$ (au voisinage de la côte) à $35^{\circ} \mathrm{C}$ (à l'intérieur du bassin). Le réseau hydrographique est représenté par l'une des principales rivières du royaume : la rivière Sebou et ses affluents: Ouargha, Beht et R'dom.

$\mathrm{Ce}$ réseau hydrographique complexe avec ses précipitations saisonnières irrégulières ainsi que ses sols argileux, présente un risque d'inondations fréquentes dans cette région. Ces précipitations se situent autour de $500 \mathrm{~mm} / \mathrm{an}$ à Kénitra dans les 20 dernières années.

Depuis 1933, il y a eu plus que 40 crues d'importance variable causant de graves dégâts aux cultures mais aussi (et surtout) aux infrastructures.

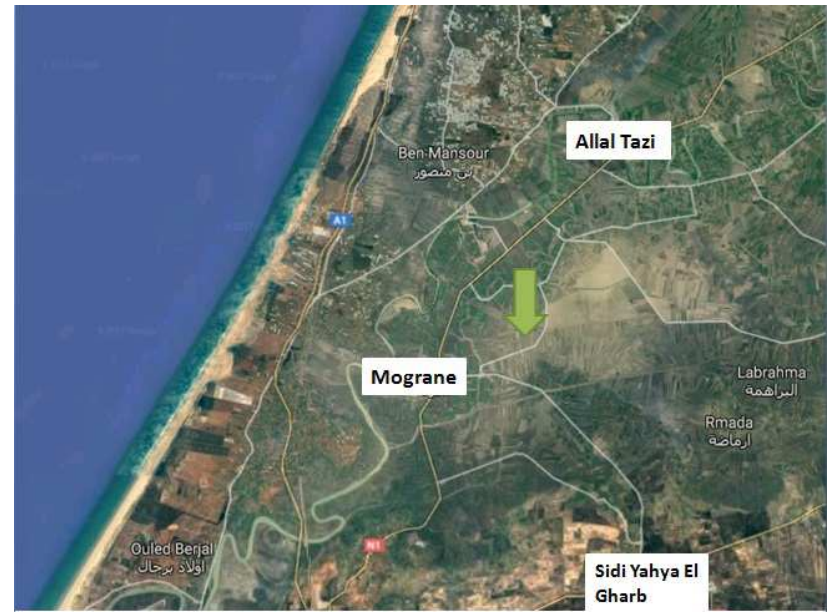

Fig. 2 : Zone d'étude (Google Maps)

Le site du projet se situe dans la région du Gharb, à $40 \mathrm{Kms}$ du NE de la ville de Kénitra (Figure 2). Il est reconnu pour ses zones inondables ainsi que ses sols gonflants (Figure 3.a/3.b), conférant ainsi à ce dernier une mauvaise portance.

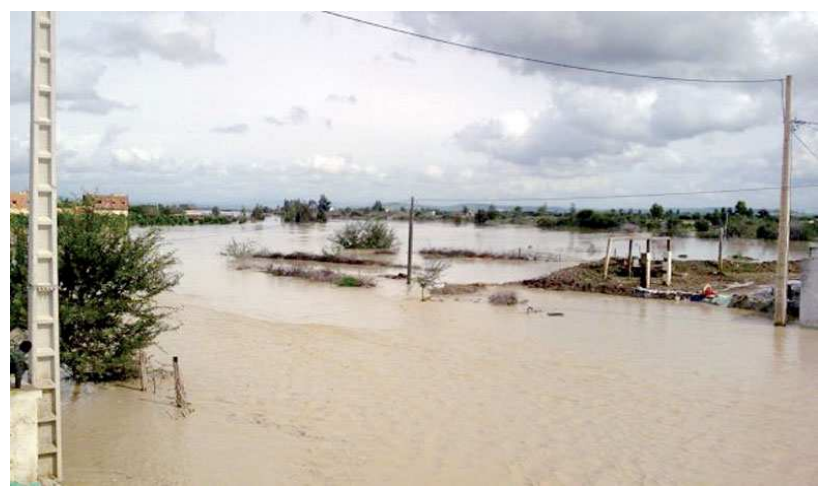

Fig. 3-a : Inondations région Kénitra (journal Le Matin /2015)

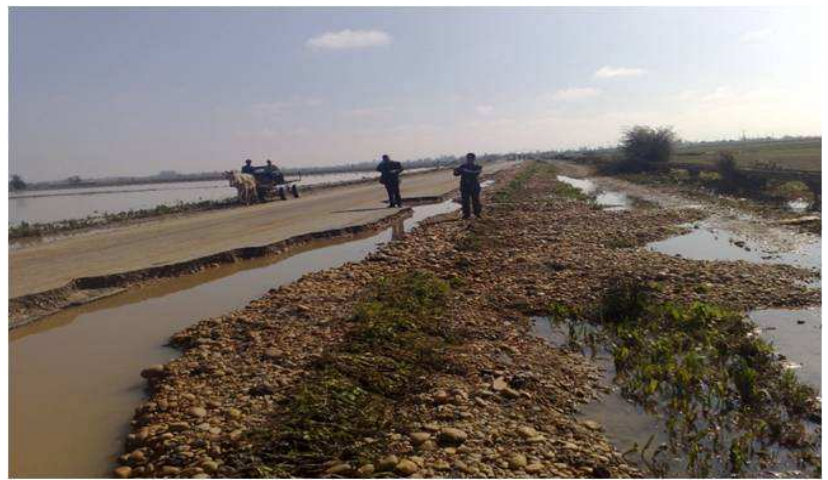

Fig. 3-b : Inondations région Kénitra 


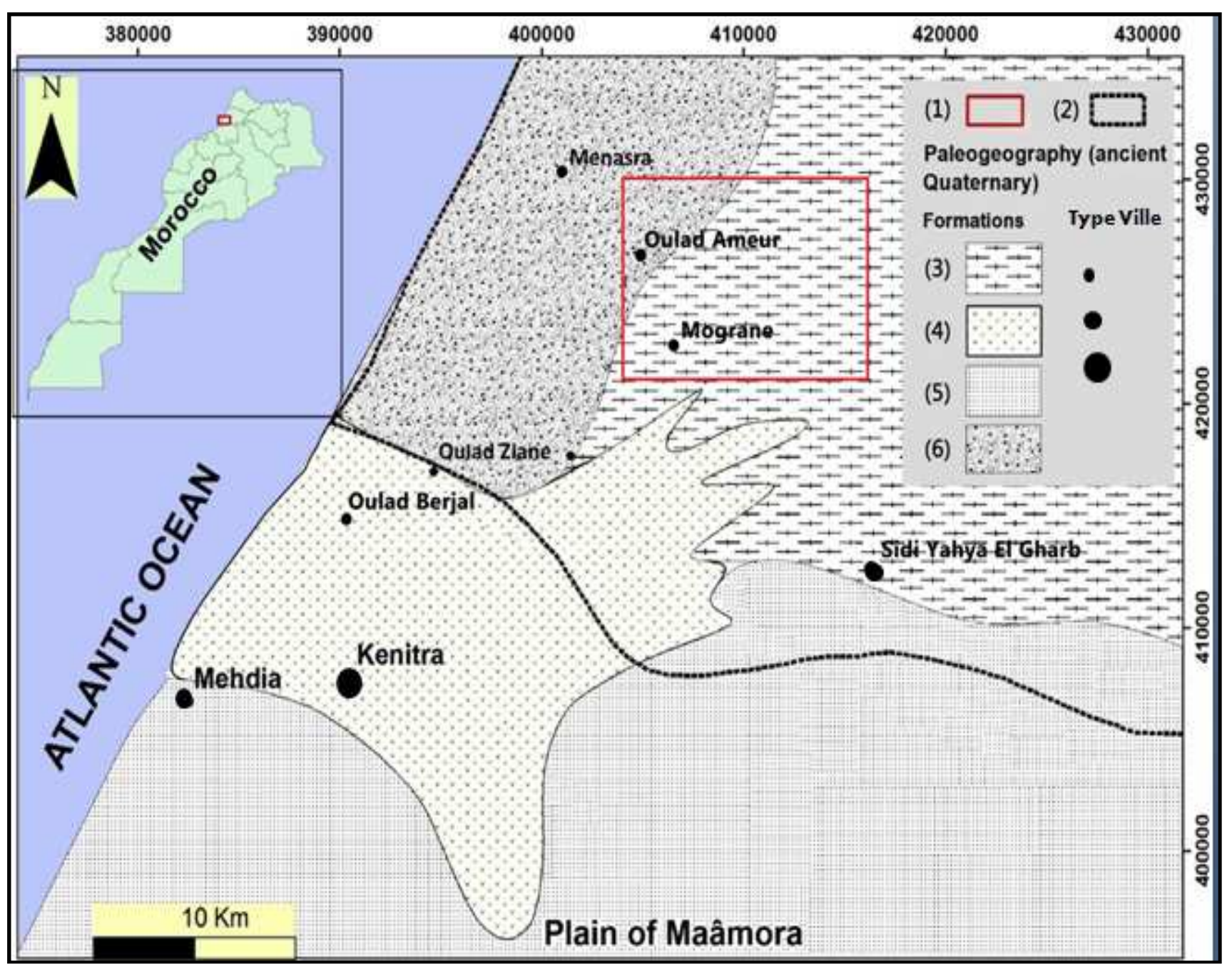

Fig. 4 : Carte de la zone d'étude dans le bassin du Gharb. 1. Limite de la zone d'étude. 2. Limite du bassin du Gharb. 3. Argile et marne de la plaine deltaïque. 4. Biocalcarénite côtière. 5. Sables et colluvions. 6 . Sables de dunes et grès.

\subsection{Cadre géologique}

La plaine du Rharb apparaît comme une cuvette qui fut subsidence depuis le Tortonien moyen et pendant tout le quaternaire. Au Sud, le glacis paléozoïque incliné de la Meseta marocaine est recouvert par des terrains tertiaires et quaternaires constitués de marnes bleues miocènes, de sables pliocènes, puis une formation rouge continental de la Maarnora, appartenant au Villafranchien. Un peu plus à l'Est, au delà de la vallée de l'oued Beht (Dar Bel Hamri), se situe le bassin des Rides prérifaines, plus complexe, constitué de terrains triasiques et jurassiques. Ce sont surtout des calcaires et des marno-calcaires, surmontés par les "grès du Zerhoun" attribués au Bathonien. Les mollasses de base du Miocène enveloppent les plis des rides calcaires [4].

La colonne stratigraphique du Gharb est composée d'un substratum d'âge paléozoïque qui intègre des sédiments et roches métamorphiques : schiste, quartzite et calcaire [17], vaincus par les dépôts argileux rouges de Trias et par les dolomites et calcaires dolomitiques du Jurassique et du Crétacé. Le Miocène qui constitue le plancher imperméable de la nappe phréatique profonde de Gharb [17], est formé par des marnes bleues. Au Pliocène, la sédimentation a une double origine: les premières transgressions sur la côte actuelle ont donné naissance à la sédimentation côtière représentée par le grès et le calcaire. Cette formation de la côte, qui peut atteindre jusqu'à $200 \mathrm{~m}$ d'épaisseur constitue l'aquifère réservoir de majeur. [5] et les transgressions du quaternaire qui ont été représentées à nouveau par une sédimentation détritique carbonatée en intercalations avec les argiles. Tout au long de cette succession sédimentaire, des argiles turbiditique se sont interstratifiés dans les marnes bleutées et gypsifères. La couverture de surface de Gharb est caractérisée par une extension des sols argileux et sableux favorables à une large gamme de cultures de céréales.

\section{Méthodologie}

Pour un meilleur résultat au traitement, une caractérisation des matériaux à traiter ainsi que des matériaux de traitement, permettra une optimisation que ce soit technique ou économique. La méthode adoptée est 
celle mentionnée dans le Guide des Traitement des Sols [6]. Nous procédons par une identification de l'échantillon du sol à traiter, ensuite une étude sur l'aptitude du traitement à effectuer sera réalisée, pour finir par une caractérisation du couple Sol-Liant et une comparaison entre les résultats obtenues avant et après traitement.

\subsection{Echantillonnage}

Après une reconnaissance géologique et géographique de la zone étudiée, on a procédé à une étude géotechnique du sol par le biais des prélèvements placés dans des sacs hermétiques afin d'éviter de les contaminer par des éléments étrangers. Ensuite ces prélèvements ont été transportés au laboratoire du Centre National d'Etudes et recherches Routière (CNER-RABAT) pour la réalisation des essais d'identification.

Le sol utilisé dans cette étude a été prélevé d'une zone des déblais au niveau de la route provinciale RP4209 entre le PK1+000 et le PK3+000.

\subsection{Les essais d'identification}

Les essais d'identification [7] ont portés sur: l'analyse granulométrique par tamisage (NM 13.1.008) [8] afin de définir la dimension du plus gros élément présent dans le sol (Dmax) ainsi que la teneur en fines. La limite d'Atterberg (NM 13.1.007) [9] et la valeur au bleu de méthylène du sol VBS (NF P 94-068) [10] pour la connaissance du niveau d'argilosité dans le sol, cette valeur avait un rôle important dans le choix du liant adopté pour le traitement.

Dans le même contexte, on a procédé à la caractérisation de l'état du sol en passant par l'état hydrique déterminée via le rapport Wn/Wopn et par l'indice portant immédiat IPI (NF P94-078) [11]. Ces caractéristiques ont été obtenues après l'exécution de l'essai Proctor selon la norme (NF P94-093) [12]. Suite aux résultats obtenus via ce dernier essai, on a pu réaliser un essai CBR (à 4 jours après immersion dans l'eau), afin de nous statuer sur la portance du sol sans traitement. Ce résultat, sera comparé avec celui obtenu par le même essai, mais cette fois avec l'ajout du liant (chaux vive).

Ensuite, un essai de stabilité des grumeaux, nous a permis dans une durée de temps rapide, d'avoir une idée sur l'aptitude du sol au traitement [13].

L'étape suivant l'essai d'aptitude au traitement, était une étude de formulation : dans ce projet, on a effectué un traitement avec un ajout de $3 \%$ de la chaux. Après échantillonnage et étuvage à $50^{\circ} \mathrm{C}$, on a procédé à la réalisation de l'essai selon les modalités en vigueur la norme (NF P94-093).

\section{$\underline{\text { Chaux }}$}

La chaux utilisée est une chaux vive CL90-Q, apportée d'une usine de production de Tlat Loulad. Les propriétés physico-chimiques de cette chaux sont récapitulées dans le tableau I.
Tableau I. Propriétés physico-chimiques de la chaux

\begin{tabular}{|c|c|}
\hline Nom & $\begin{array}{c}\text { Caractéristiques de la } \\
\text { chaux }\end{array}$ \\
\hline Apparence & Poudre de couleur blanche \\
\hline $\mathrm{SiO} 2$ & $0,6 \%$ \\
\hline $\mathrm{Al} 2 \mathrm{O} 3$ & $0,2 \%$ \\
\hline $\mathrm{Fe} 2 \mathrm{O} 3$ & $0,2 \%$ \\
\hline $\mathrm{MgO}$ & 0,3 \\
\hline $\mathrm{CaO}$ total & $\geq 90 \%$ \\
\hline Réactivité $\mathrm{T} 60$ & $\leq 4$ minutes \\
\hline
\end{tabular}

\section{Résultats et discussion}

\subsection{Sans traitement :}

Les propriétés physiques et mécaniques de ce sol étudié sont récapitulées dans le tableau II.

Tableau II. Propriétés physiques et mécaniques du sol

\begin{tabular}{|c|c|}
\hline Les essais effectués sur le sol & Résultats \\
\hline Particules $\geq 50 \mathrm{~mm}$ & 0 \\
\hline Particules $\leq 80 \mathrm{~mm}$ & $\geq 90 \%$ \\
\hline Limite de liquidité & 61 \\
\hline Limite de plasticité & 30 \\
\hline Indice de plasticité & 31 \\
\hline Valeur au bleu de méthylène (VBS) & 6 \\
\hline Teneur en eau naturelle (Wn) & $23,53 \%$ \\
\hline Teneur en eau à l'optimum (Wopn) & $22,81 \%$ \\
\hline Densité sèche optimale & 1,55 \\
\hline Indice CBR (4jrs) & 2 à 3 \\
\hline
\end{tabular}

Compte tenue des résultats obtenus lors de notre étude, on constate que le sol étudié dans cet article est de classe A3 selon la classification GMTR [14]. Une classe dont l'argilosité est moyennement à très argileuse $(\mathrm{IP}=31)$.

Cette conclusion reflète une mauvaise réaction du sol lors du compactage. Les résultats obtenus après la réalisation des essais Proctor, montrent une densité sèche maximale de 1,55 pour une teneur en eau optimale (Wopn) Wopn $=22,81 \%$ (Figure 5) et un indice portant immédiat (IPI) entre 8 et 14 . Après traitement cette valeur diminue nettement pour atteindre une valeur de 1.36 de densité sèche maximale pour une teneur en eau optimale (Wopn) Wopn =20,25\% (figure 7). 


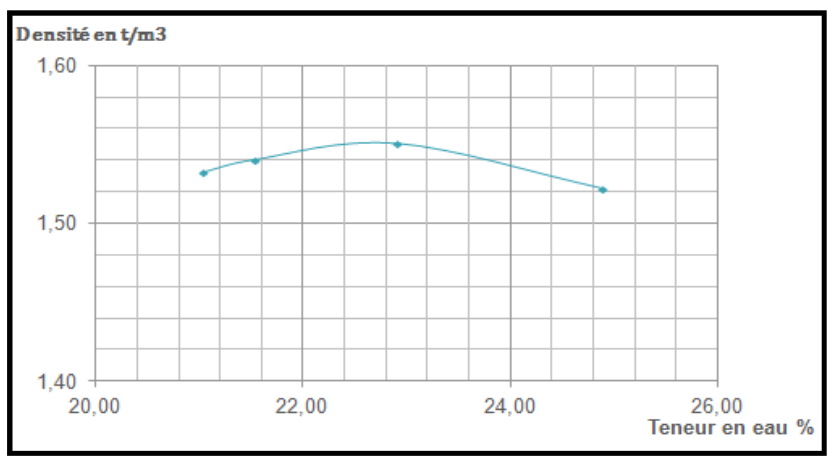

Fig. 5 : Courbe PROCTOR normal (sans traitement)

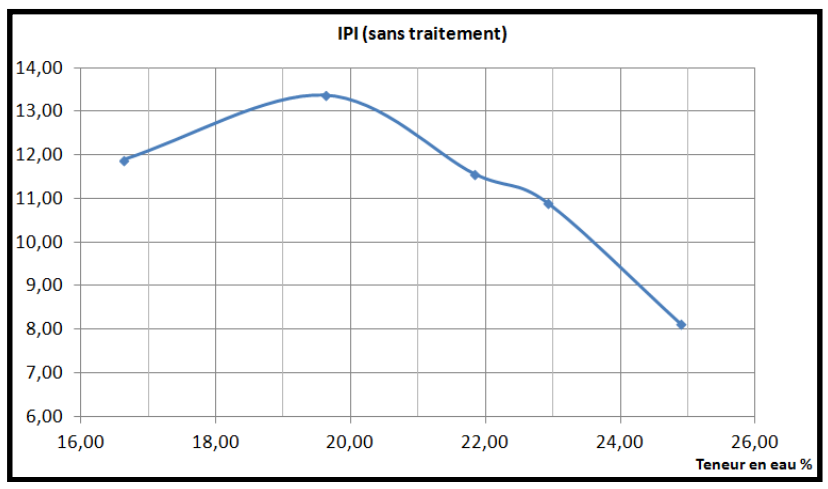

Fig. 6 : Courbe IPI normal (sans traitement)

L'indice $\mathrm{CBR}_{\mathrm{i}}$ (à $4 \mathrm{jrs}$ ) est $\leq 4$, ce qui (selon le catalogue des structures types de chaussées neuves CSTCN [15]) confère au sol une portance St0 (portance faible).

\subsection{Avec traitement (de $3 \%$ de chaux) :}

Après un premier essai de traitement à $3 \%$ de la chaux, la densité sèche optimale décroit à 1,36 pour une teneur en eau optimale (Wopn) de 20,25). L'indice immédiat portant (IPI) s'est amélioré entre un intervalle de 12 à 21.

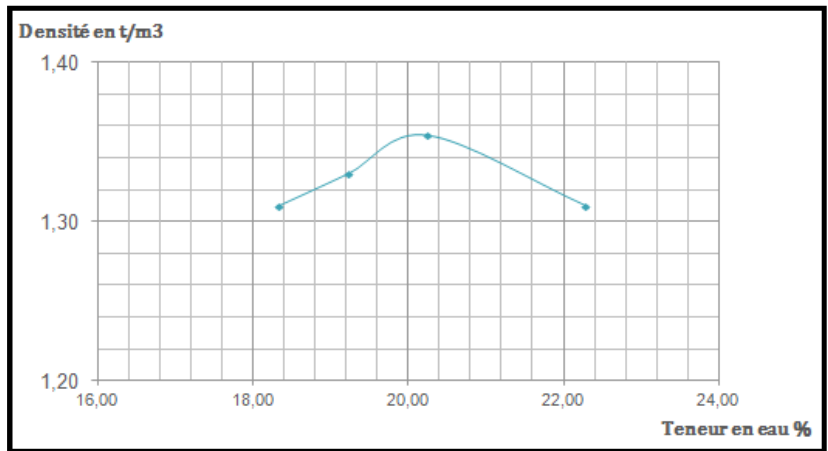

Fig. 7 : Courbe PROCTOR normal (avec traitement)

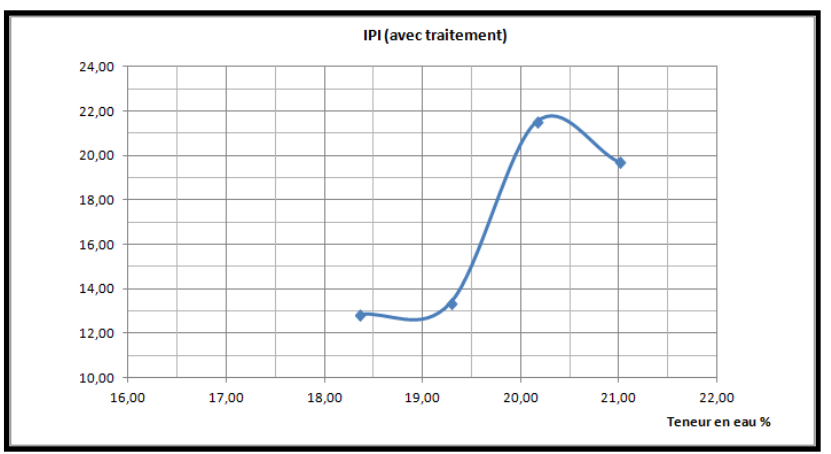

Fig. 8 : Courbe IPI normal (avec traitement)

Une comparaison entre IPI sans traitement (Figure 6) et IPI avec traitement (Figure 8), a permis de montrer une nette amélioration de la portance du sol, ceci est dû principalement à l'effet de la floculation obtenu par l'ajout de la chaux [16].

D'autres essais ont été effectués sur le sol traité pour définir l'Indice de Plasticité. Il en est ressort comme résultat pour IP (avec traitement à la chaux), une valeur de $14 \%$. On remarque que la chaux a un impact important sur la plasticité du sol, impliquant une diminution de la sensibilité de la marne argileuse, soit une réduction la sensibilité à l'eau et du degré du gonflement [16].

L'indice $\mathrm{CBR}_{\mathrm{i}}$ (à 4jrs) (avec traitement à la chaux) a augmenté jusqu'à atteindre une valeur de 14, ce qui (selon le catalogue des structures types de chaussées neuves CSTCN) améliorant ainsi la portance du sol (St2).

\section{Conclusion}

L'étude des marnes argileuses des zones inondables de la région de Kénitra ont de mauvaises propriétés géotechniques. L'étude a permis l'identification des matériaux de la zone du projet, de classer ces marnes et de déterminer leur comportement mécanique.

Ces marnes sont de classe A3 selon la classification GMTR avec une argilosité moyenne à très forte $(\mathrm{IP}=31)$. Ces marnes s'avèrent incapables de supporter toute charge importante.

Le traitement de ces sols par l'addition de la chaux au laboratoire a nettement amélioré leur qualité. En effet, une diminution nette de la densité sèche du matériau qui passe de 1.55 à 1,36, soit une diminution de $12.25 \%$; une augmentation de la valeur de l'IPI qui évolue de 13 à 22, soit une augmentation de $69.26 \%$ et enfin une augmentation importante de la valeur du $\mathrm{CBR}_{\mathrm{i}}$ à $4 \mathrm{j}$ de 3 à 14 , soit un gain considérable qui s'élève à $366.67 \%$.

Ainsi, les résultats prometteux obtenus ont démontré l'aptitude du traitement avec la chaux à améliorer la portance de ces marnes.

\section{Références}

1. IDRRIM, Institut Des Routes, des Rues et des Infrastructures pour la Mobilité, Enseignements de 
TerDOUEST- Propositions de compléments au Guide Traitement des Sols- (Décembre 2015).

2. Magazine «Routes»: Le traitement des sols à la chaux et/ou aux liants hydrauliques pour l'exécution des remblais et des couches de forme. Rubrique: Documentation technique. p.7-14, 98. (Septembre 2004).

3. Ministère de l'Agriculture et de la Réforme Agraire. Royaume du Maroc Le Périmètre du Gharb; ORMVAG (Office Régionale de Mise en Valeur Agricole du Gharb). (1997).

4. Mohamed EL Bouhaddioui, Abdelaziz Mridekh, Malika Kili, Bouabid El Mansouri, El Houssine EL Gasmi, Bouchaib Magrane. Electrical and well log study of the Plio-Quaternary deposits of the southern part of the Rharg Basin, northern Morocco. Journal of African Earth Sciences. (2016).

5. Combe, M., Bassin du Rharb Maâmora. In ressources en eau du Maroc. Tome2. Notes et Mem, Serv, Geol, Maroc, 231, pp. 93 e128. (1975)

6. Guide technique SETRA-LCPC. Traitement des sols à la chaux et/ou aux liants hydrauliques-Application à la réalisation des remblais et des couches de forme (GTS). (janvier 2000).

7. AFNOR NF P 94-100: Sols: Reconnaissance et essais. Matériaux traités à la chaux et/ou aux liants hydrauliques- Essais d'évaluation de l'aptitude d'un sol au traitement. (1999).

8. IMANOR NM 13.1.008: Analyse granulométrique des sols par tamisage. (1998).

9. IMANOR NM 13.1.007: Essai d'identification: Détermination des limites d'ATTERBERG- Limite de plasticité au rouleau- Limite de liquidité à la coupelle. (1998).

10. AFNOR NF P94-068: Sols: Reconnaissance et essais. Mesure de la capacité d'absorption de bleu de méthylène d'un sol ou d'un matériau rocheux par l'essai à la tâche. (1998).

11. AFNOR NF P94-078: Sols: Reconnaissance et essais. Indice CBR après immersion- Indice CBR immédiat- Indice Portant Immédiat. (1997).

12. AFNOR NF P94-093: Sols: Reconnaissance et essais. Détermination des références de compactage d'un matériau. (1999).

13. Centre de Recherches Routières: Mode opératoire des essais en laboratoire pour l'évaluation « de l'aptitude d'un sol à la stabilisation immédiate à la chaux ». Méthode de Mesure CRR-MF 33/72.

14. Direction des routes et de la circulation routière \& Laboratoire Public d'Essais et d'Etudes (MAROC) : Guide marocain pour les terrassements routiers «GMTR» (2002).

15. Direction des Routes et de la Circulation Routière \& Laboratoire Public d'Essais et d'Etudes (MAROC): Catalogue des structures types de chaussées neuves (1995).

16. CIMBETON, Centre d'information sur le ciment et ses applications: Traitements des sols aux liants hydrauliques. Collection technique. (Novembre 2009).

17. MICHARD A. Eléments de la géologie marocaine. Notes et Mémoires, Service Géologique du Maroc, $\mathrm{n}^{\circ} 252,420 \mathrm{pp}, 1976$ 